\title{
THE EMBEDDABILITY OF RING AND SEMIGROUP AMALGAMS IS UNDECIDABLE
}

\author{
MARCEL JACKSON
}

(Received 1 April 1999; revised 16 June 2000)

Communicated by D. Easdown

\begin{abstract}
We prove that there is no algorithm to determine when an amalgam of finite rings (or semigroups) can be embedded in the class of rings or in the class of finite rings (respectively, in the class of semigroups or in the class of finite semigroups). These results are in marked contrast with the corresponding problems for groups where every amalgam of finite groups can be embedded in a finite group.
\end{abstract}

2000 Mathematics subject classification: primary 16B99, 20M99; secondary 20M25, $20 \mathrm{M} 05$.

\section{Introduction}

Let $K$ be a class of algebras of some fixed type $\mathscr{L}$ (for example: rings, groups, semigroups, heaps, finite rings). Let $\left\{\mathbf{S}_{i}: i \in I\right\}$ be a set of members of $K$ indexed by the set $I$ such that for some type $\mathscr{L}$ algebra $\mathrm{U}$ there are injective homomorphisms $\phi_{i}: \mathbf{U} \rightarrow \mathbf{S}_{\mathbf{i}}$. This collection of algebras and mappings is called a (finite) $K$ amalgam and is denoted by $\left[\left\{\mathbf{S}_{\mathbf{i}}: i \in I\right\} ; \mathbf{U} ;\left\{\phi_{i}: i \in I\right\}\right]$ or more briefly $\left[\mathbf{S}_{\mathbf{i}} ; \mathbf{U} ; \phi_{i}\right]$ or even simply $\left[\mathbf{S}_{\mathrm{i}} ; \mathbf{U}\right]$. Less formally, a $K$ amalgam may be viewed as a collection of algebras from $K$ (the $\mathrm{S}_{\mathrm{i}}$ ) each sharing a common subalgebra from $K$ (the algebra $\mathbf{U}$ ). The algebra $\mathbf{U}$ is known as the core of the amalgam.

An embedding of a $K$ amalgam $\left[\mathbf{S}_{\mathrm{i}} ; \mathbf{U} ; \phi_{i}\right]$ is a set of injective homomorphisms $\left\{v_{i}: i \in I\right\}$ with $v_{i}: \mathbf{S}_{\mathbf{i}} \rightarrow \mathbf{T}$ for some type $\mathscr{L}$ algebra $\mathbf{T}$ so that for $s \in \mathbf{S}_{\mathbf{i}}$ and $t \in \mathbf{S}_{\mathbf{j}}$, $v_{i}(s)=v_{j}(t)$ if and only if $i=j$ and $s=t$ or there is a $u \in \mathbf{U}$ such that $\phi_{i}(u)=s$ and $\phi_{j}(u)=t$.

The fundamental question to be asked concerning a $K$ amalgam is the following:

(c) 2000 Australian Mathematical Society $0263-6115 / 2000 \$ A 2.00+0.00$ 
QUESTION 1.1. Given a finite $K$ amalgam $\mathbf{A}=\left[\mathbf{S}_{\mathbf{i}} ; \mathbf{U}\right]$, is $\mathbf{A}$ embeddable in an algebra from $K$ ?

The classes $K$ which we will be primarily concerned with in this paper are the class of all semigroups, the class of all rings, the class of finite semigroups and the class of finite rings. For the class of all groups and the class of finite groups, Question 1.1 has a remarkably simple solution: the answer is 'always', Schreier [13]. For semigroups and rings however this is not the case. Consider the following pair of semigroups:

\begin{tabular}{l|llll}
$\cdot$ & 0 & 1 & 2 & 3 \\
\hline 0 & 0 & 0 & 0 & 0 \\
1 & 0 & 0 & 0 & 1 \\
2 & 0 & 0 & 0 & 1 \\
3 & 0 & 1 & 1 & 3
\end{tabular}

\begin{tabular}{c|cccc}
$\cdot$ & 0 & 1 & 2 & 4 \\
\hline 0 & 0 & 0 & 0 & 0 \\
1 & 0 & 0 & 0 & 2 \\
2 & 0 & 0 & 0 & 2 \\
4 & 0 & 2 & 2 & 4
\end{tabular}

The two semigroups share a common three element semigroup with zero multiplication and so we may consider them as a semigroup amalgam. However this amalgam is not embeddable in any semigroup since in that case we would have

$$
3 \cdot(1 \cdot 4)=3 \cdot 2=1 \text { and }(3 \cdot 1) \cdot 4=1 \cdot 4=2
$$

That is, associativity fails in any groupoid in which the amalgam is embeddable (the first example of this kind was found by Kimura; [1, Volume II, page 139]). It is clear that a semigroup amalgam $\mathbf{A}$ determines a partial groupoid in a natural way but the example above shows that this is not necessarily a partial semigroup in the sense that we do not necessarily have $(x y) z=x(y z)$ whenever both sides of this expression are defined.

Question 1.1 for rings and semigroups has consequently been the subject of a substantial quantity of work and several books on semigroup theory contain a chapter devoted to it and associated concepts. The question may of course be restated as a decision problem (which we now formulate specifically in terms of rings and semigroups):

PROBlem 1.2. Determine if a finite ring (semigroup) amalgam $A=\left[\mathbf{S}_{i} ; \mathbf{U}\right]$ is embeddable in ring (or semigroup).

More generally we may state the following problems:

PROBlem 1.3. (i) Given a finite ring (semigroup) amalgam $\mathbf{A}=\left[\mathbf{S}_{\mathbf{i}} ; \mathbf{U}\right]$ of rings (semigroups) from a class $K$, determine if $\mathbf{A}$ is embeddable in a ring (semigroup) from $K$.

(ii) Given a finite ring (semigroup) amalgam $A=\left[\mathbf{S}_{\mathbf{i}} ; \mathbf{U}\right]$, determine if $A$ is embeddable in a ring (semigroup) from a class $\mathrm{K}$. 
We will call Problem 1.3 (i) and Problem 1.3 (ii) (within the class of rings or semigroups) the strong decision problem for amalgam embeddability in $K$ and the weak decision problem for amalgam embeddability in $K$ respectively. Clearly Problem 1.2 for rings (semigroups) is simply the strong decision problem for amalgam embeddability in the class of all rings (semigroups). Note that a negative answer to the strong decision problem for a class $K$ implies a negative answer to the weak decision problem for $K$. In terms of decidability and undecidability these problems will coincide for some classes. For example if $K$ is a variety (that is an equationally defined class) then any ring (semigroup) amalgam containing a ring (semigroup) not from $K$ (that is, not satisfying one of the defining identities of $K$ ) clearly is not embeddable in a member of $K$. Recent results of Kublanovsky and Sapir [8] can be used to show that the strong and weak decision problems for embeddability of ring (semigroup) amalgams in the class of finite rings (semigroups) are undecidable (see Theorem 3.3 below). The main result of this paper is the following theorem.

THEOREM 1.4. Let $\mathbf{A}=\left[\mathbf{S}_{\mathrm{i}} ; \mathbf{U}\right]$ be a finite ring (semigroup) amalgam. Then there is no algorithm to decide whether $\mathbf{A}$ is embeddable in a ring (semigroup) or a finite ring (finite semigroup). That is, the strong and weak decision problems for embeddability of amalgams in the class of rings (semigroups) and in the class of finite rings (finite semigroups) are undecidable.

In particular Problem 1.2 is undecidable. We note that there are several important classes for which the corresponding problem has a very different solution. We have seen that any (finite) group amalgam can be embedded in a finite group. Similarly Hall has shown that any (finite) amalgam of inverse semigroups can be embedded in an inverse semigroup [2], however this is not necessarily finite (see [5, page 309] for an example, due to $\mathrm{C}$. $\mathrm{J}$. Ash, of an inverse semigroup amalgam not embeddable in a finite semigroup). Interestingly, we will show in Corollary 3.2 that the weak decision problem for inverse semigroups and finite inverse semigroups is undecidable. The class of subsemigroups of inverse semigroups has a decidable membership problem; see [1, Volume II] for a description due to B. Schein. However if $K$ is a class with undecidable membership problem then the weak decision problem for amalgam embeddability in $K$ must also be undecidable since individual rings (semigroups) can be considered, trivially, as amalgams by themselves ([S; $\mathbf{S}]$ in our notation). A shining example of such a class is the class of subsemigroups of completely 0simple semigroups which was shown to have this property by Kublanovsky (see [3]). Naturally, this type of argument does not apply to the strong decision problem for amalgam embeddability.

A generalization of amalgam embeddability is weak amalgam embeddability (see [5]). If $\mathbf{A}$ is a ring (semigroup) amalgam $\left[\mathbf{S}_{\mathbf{i}} ; \mathbf{U} ; \phi_{i}\right]$ then we will say $\mathbf{A}$ is weakly embeddable in a ring (semigroup) $\mathbf{T}$ if for each $i$ there are injective homomorphisms 
$v_{i}: S_{\mathbf{i}} \rightarrow \mathbf{T}$ such that for every $u \in \mathbf{U}, \phi_{i}(u)=s$ and $\phi_{j}(u)=t$ imply $v_{i}(s)=v_{j}(t)$. So any embedding of an amalgam is a weak embedding but not every weak embedding is an embedding. We can replace 'embeddable' with 'weakly embeddable' in Problem 1.3 (i) and (ii) and call the respective decision problems the strong decision problem for weak amalgam embeddability in $K$ and the weak decision problem for weak amalgam embeddability in $K$. It is conceivable that a class $K$ has an undecidable (strong or weak) decision problem for amalgam embeddability but a decidable (strong or weak) decision problem for weak amalgam embeddability (or vice versa). We will show that this is not the case for the class of all rings (semigroups) and the class of finite rings (semigroups).

THEOREM 1.5. The strong and weak decision problems for weak embeddability of ring (semigroup) amalgams in the class of all rings (semigroups) and in the class of finite rings (finite semigroups) are undecidable.

Let $\mathbf{S}$ be a semigroup and $\mathbb{Z}_{2}$ be the field of two elements, $\{0,1\}$. Then the universe of the semigroup ring $\mathbb{Z}_{2}[\mathbf{S}]$ is the set of all functions $f: S \rightarrow\{0,1\}$ which map only finitely many elements of $\mathbf{S}$ to 1 . The addition on $\mathbb{Z}_{2}[\mathbf{S}]$ is pointwise and the multiplication is defined by $f g(s)=\sum_{s_{i} s_{j}=s} f\left(s_{i}\right) g\left(s_{j}\right)$. There is a natural embedding of every semigroup $\mathbf{S}$ into the multiplicative semigroup of the semigroup ring $\mathbb{Z}_{2}[\mathbf{S}]$ which sends an element $s$ to the function $f_{s}$ defined by $f_{s}(t)=1$ if $s=t$ and 0 otherwise. Also if $\mathbf{S}$ is a subsemigroup of a semigroup $\mathbf{T}$, then by considering those elements of $\mathbb{Z}_{2}[\mathbf{S}]$ which are functions sending all elements $t \in \mathbf{T} \backslash \mathbf{S}$ to 0 we have that the semigroup ring $\mathbb{Z}_{2}[\mathbf{S}]$ is a subring of $\mathbb{Z}_{2}[\mathbf{T}]$. These facts enable one to translate many semigroup embedding problems into ring embedding problems. This will also be true of amalgam embeddability.

Given a semigroup amalgam $\mathbf{A}=\left[\mathbf{S}_{\mathbf{i}} ; \mathbf{U}\right]$ we can construct the ring amalgam $\mathbb{Z}_{2}[\mathbf{A}]=\left[\mathbb{Z}_{2}\left[\mathbf{S}_{\mathbf{i}}\right] ; \mathbb{Z}_{2}[\mathbf{U}]\right]$. The amalgam $\mathbf{A}$ can be embedded into the multiplicative semigroup amalgam of $\mathbb{Z}_{2}[\mathbf{A}]$ as a 'sub-amalgam' in the natural way. If $\mathbf{A}$ is (weakly) embeddable in $\mathbf{T}$, then $\mathbb{Z}_{2}[\mathbf{A}]$ is (weakly) embeddable in $\mathbb{Z}_{2}[\mathbf{T}]$ (which is finite if and only if $\mathbf{T}$ is). Furthermore, if $\mathbb{Z}_{2}[\mathbf{A}]$ is (weakly) embeddable in a ring or finite ring $\mathbf{R}$ then the amalgam $\mathbf{A}$ (which is a 'sub-amalgam' of the multiplicative semigroup amalgam of the ring amalgam $\mathbb{Z}_{2}[\mathbf{A}]$ ) is (weakly) embeddable in the multiplicative semigroup of $\mathbf{R}$. Thus it will suffice to prove Theorem 1.4 and Theorem 1.5 in the case of semigroups.

Theorem 1.4 and Theorem 1.5 admit a relatively simple proof in the style of several other recent undecidability results concerning embedding problems of finite semigroups and general algebras: $[3,6,8,9,11]$. In particular, the results in [8] can be modified to give Theorem 3.3 in this paper. Common to all of these papers are proofs roughly of the following format: for every partial group $\mathbf{G}$ a finite semigroup (or algebra) $\mathbf{S}$ is constructed such that the answer to the relevant embedding problem 
is 'yes' for $\mathbf{S}$ if and only if $\mathbf{G}$ is embeddable in a group. Since the embeddability of finite partial groups in the class of all groups or the class of finite groups is undecidable (see [7, Connection 2.2]), the result follows.

After the above results were obtained (in 1997) the author was informed by Mark Sapir that he had earlier obtained Theorem 1.4 using the evidently different method of Minsky machines. In fact Sapir shows that there exists an amalgam of two finite semigroups whose amalgamated free product has an undecidable word problem [12]. While this surprising result was not obtained by the author of this paper, the proof we give here of Theorem 1.4 avoids any technicalities associated with the Minsky machine encoding used in [12] and allows the introduction of some significant bounds on the size of the core semigroup and also on one of the semigroups involved in the amalgam (a finite bound on the cardinality of the entire amalgam would obviously imply decidability of the corresponding embedding problem since there would be only finitely many amalgams to consider). The proof of the undecidability of the strong decision problem for amalgam embeddability in the class of finite semigroups presented in [12] is similar to the one given here.

\section{Preliminaries}

Throughout this paper we will adopt the notation that if $\mathbf{S}$ is a semigroup with identity element, then $\mathbf{S}^{\mathbf{1}}$ denotes simply $\mathbf{S}$ and if $\mathbf{S}$ is a semigroup without identity element, then $\mathbf{S}^{\mathbf{1}}$ denotes the semigroup obtained from $\mathbf{S}$ by adjoining an identity element. When considering a semigroup (or any other kind of algebraic structure) $\mathbf{S}$, by $S$ we will mean the underlying set or universe on which the operation of $\mathbf{S}$ is defined (or partially defined, as the case may be).

On any semigroup $\mathbf{S}$ we can define the following equivalence relations

$$
\begin{aligned}
& \mathscr{L}^{\mathrm{S}}=\left\{(a, b): \exists x, y \in S^{1} \text { such that } x a=b, y b=a\right\}, \\
& \mathscr{R}^{\mathrm{S}}=\left\{(a, b): \exists x, y \in S^{1} \text { such that } a x=b, b y=a\right\}, \\
& \mathscr{J}^{\mathrm{S}}=\left\{(a, b): \exists w, x, y, z \in S^{1} \text { such that } w a x=b, y b z=a\right\}, \\
& \mathscr{H}^{\mathrm{s}}=\mathscr{L} \cap \mathscr{R}, \quad \mathscr{D}^{\mathrm{S}}=\mathscr{L} \circ \mathscr{R}=\mathscr{R} \circ \mathscr{L} .
\end{aligned}
$$

When there is no confusion as to what semigroup a particular relation is being defined on, the superscripts of these relations will be dropped. These five equivalence relations are known as Green's relations and are fundamental concepts in the study of semigroups. We will denote by $L_{a}$ (respectively $R_{a}, H_{a}, J_{a}, D_{a}$ ) the equivalence class of $\mathscr{L}$ (respectively $\mathscr{R}, \mathscr{H}, \mathscr{J}, \mathscr{D}$ ) containing $a$.

Two important results concerning Green's relations are the following. Proofs will be omitted since they are well known and can be found in almost any semigroup textbook (see [1] or [5] for example). 
LEMMA 2.1 (Green). Let $a$ and $b$ be two $\mathscr{R}$ equivalent elements of a semigroup $\mathbf{S}$ and let $s, t \in S^{1}$ be such that $a s=b$ and $b t=a(s, t$ exist by the definition of $\mathscr{R})$. Then the mappings given by $x \mapsto x s$ and $y \mapsto y t$ for $x \in L_{a}, y \in L_{b}$ are $\mathscr{R}$-class preserving, mutually inverse, injective mappings from $L_{a}$ to $L_{b}$ and from $L_{b}$ to $L_{a}$ respectively.

Recall that an element $a$ of a semigroup $\mathbf{S}$ is regular if there is an $x$ such that $\operatorname{axa}=a$.

LEMMA 2.2. (i) If a $\mathscr{D}$-class $D$ of a semigroup $\mathbf{S}$ contains a regular element then every element of $D$ is regular and $D$ is called a regular $\mathscr{D}$-class of $\mathbf{S}$.

(ii) If a $\mathscr{D}$-class $D$ of a semigroup $\mathbf{S}$ is regular then every $\mathscr{L}$-class and every $\mathscr{R}$-class in $D$, contains an $\mathscr{H}$-class that is a subgroup of $\mathbf{S}$.

The following concept due to Sapir plays an important role in [8] and [11].

DEFINITION 2.3. A split system is a triple of sets $(A, B, C)$ with an associated operation $A \times B \rightarrow C$. An embedding of a split system into a semigroup $\mathbf{S}$ is a triple $(i, j, k)$ of injective maps $j: A \rightarrow S, k: B \rightarrow S$ and $l: C \rightarrow S$ and $j(a) k(b)=l(a b)$, for each $a \in A$ and $b \in B$.

A variation on split systems called split pairs play an analogous role in [6].

As mentioned above, the proof of Theorem 1.4 will use the undecidability of the embedding problem of finite partial groups in the class of all groups and the class of finite groups (see [7, Connection 2.2]). By a partial group we will mean a set with an element 1 and with a partial binary operation which is, where defined, associative and satisfies $1 x=x 1=x$ and is such that if $g h=g k$ or $h g=k g$ then $h=k$. A symmetric partial group (see [8]) is a partial group $\mathbf{G}$ with the property that for every $g \in G$ there is a unique $g^{\prime} \in G^{\prime}$ such that $g g^{\prime}=g^{\prime} g=1$. For any finite partial group we may construct a symmetric extension $\mathbf{G}^{\prime}$ of $\mathbf{G}$ which is a symmetric partial group containing $\mathbf{G}$ such that for every $g \in G^{\prime}$, either $g$ or $g^{\prime}$ is contained in the set $G$. This last condition ensures that there are only finitely many possible symmetric extensions and they may be effectively listed. It is also clear that if $\mathbf{G}$ is embeddable in a group, then there is a symmetric extension of $\mathbf{G}$ that is embeddable in a group, since every group may be considered as a symmetric partial group (where the 'partial' operation is defined everywhere). Thus the problem of determining whether a finite symmetric partial group is embeddable in a group or in a finite group is undecidable also; otherwise we would have the following algorithm for checking embeddability of finite partial groups. Construct all symmetric extensions of the given partial group and check if any are embeddable in a group or finite group. If the answer is yes, then the original partial group is embeddable in the same group. If the answer is no, then 
the original partial group is not embeddable in a group or a finite group (this would of course be a rather slow algorithm, but an algorithm nevertheless).

The following definition first appeared in [3].

DEFINITION 2.4. Let $\mathbf{G}$ and $\mathbf{G}^{\prime}$ be partial groups such that $\mathbf{G}$ is embedded in $\mathbf{G}^{\prime}$. For each $i=0,1,2, \ldots$, let $G^{i}$ be the subset of the universe of $\mathbf{G}^{\prime}$ defined as follows: $G^{0}=\{1\}$ (the identity element), $G^{1}=G, G^{i+1}=G^{i} G$. Then for $k \geq 2$, the partial group $\mathbf{G}^{\prime}$ is an extension of rank $k$ of $\mathbf{G}$ if and only if

(1) $G^{\prime}=\bigcup_{i=0}^{k} G^{i}$,

(2) for every pair of positive integers $i, j$ with $i+j \leq k$ and every pair of elements $x \in G^{i}, y \in G^{j}$, the product $x y$ exists and is contained in $G^{i+j}$,

(3) if $i+j>k$ and $x \in G^{i} \backslash G^{i-1}, y \in G^{j} \backslash G^{j-1}$ then the product $x y$ is not defined,

(4) if $i+j+l \leq k$ and $x \in G^{i}, y \in G^{j}, z \in G^{l}$, then $(x y) z$ and $x(y z)$ are defined and equal,

(5) for $f, g, h \in G^{\prime}, f g=f h$ or $g f=h f$ if and only if $g=h$.

For all arguments to follow we will take $\mathbf{G}_{4}$ to be an extension of rank four of a finite symmetric partial group $\mathbf{G}_{1}$ and $G_{i}$ will be used to denote the set $\bigcup_{j=0}^{i} G^{j}$ for $i \leq 4$.

A group $\mathbf{H}$ can be viewed trivially as an extension of arbitrary rank of itself. So if a symmetric partial group $\mathbf{G}$ is embeddable in a group $\mathbf{H}$, then for every $k$ there is an extension of rank $k$ of $\mathbf{G}$ that is also embeddable in $\mathbf{H}$, and since there are only finitely many possible extensions of rank $k$ of a finite partial group, all of which can be effectively listed, we have the following lemma.

LEMMA 2.5 ([3]). The problem of determining whether or not an extension of rank $k$ of a partial group is embeddable in a group or in a finite group is undecidable.

With every extension $\mathbf{G}^{\prime}$ of rank two of a partial group $\mathbf{G}$ we may construct an associated split system

$$
\left(\{a\} \times G \times\{b\},\{b\} \times G \times\{c\},\{a\} \times G^{\prime} \times\{c\}\right) .
$$

Where the operation is defined as follows

$$
(a, g, b) \cdot(b, h, c)=(a, g h, c)
$$

where $g h$ is the product of $g$ with $h$ in $\mathbf{G}^{\prime}$.

We now state a fundamental lemma concerning split systems constructed in this way. This is proved in [8] and [11]. An analogous result for the related 'split pairs' is implicit in [6]. 
LEMMA 2.6. Let $\left(\{a\} \times G \times\{b\},\{b\} \times G \times\{c\},\{a\} \times G^{\prime} \times\{c\}\right)$ be the split system associated with $\mathbf{G}^{\prime}$, an extension of rank 2 of a finite symmetric partial group $\mathbf{G}$. Then $\mathbf{G}^{\prime}$ is embeddable in a group (finite group) if and only if there is an embedding $(j, k, l)$ of $\left(\{a\} \times G \times\{b\},\{b\} \times G \times\{c\},\{a\} \times G^{\prime} \times\{c\}\right)$ into a semigroup (finite semigroup) $\mathrm{T}$ such that image of $\{a\} \times G \times\{b\}$ under $j$ and $\{b\} \times G \times\{c\}$ under $k$ lie within $\mathscr{H}^{\mathrm{T}}$-classes. An embedding of a split system in this way is called an $\mathscr{H}$-embedding.

DEFINITION 2.7. For any group $\mathbf{H}$, the Brandt semigroup $\mathbf{B}_{\mathbf{n}}(\mathbf{H})$ is the semigroup of elements of the form $\{0\} \cup\{(i, g, j): g \in H, 0<i, j<n+1\}$ with multiplication $(i, g, j) \cdot(k, h, l)=(i, g h, l)$ if $j=k$ and 0 otherwise.

More information on Brandt semigroups and their important role in semigroup theory can be found in [1] or [5]. Despite the apparently simple structure of the Brandt semigroups, in [3] it is shown that the set of finite subsemigroups of Brandt semigroups is a non recursive set.

We now define a finite semigroup $\mathbf{S}\left(\mathbf{G}_{1}, \mathbf{G}_{4}\right)$ corresponding to any finite extension $\mathbf{G}_{\mathbf{4}}$ of rank four of a symmetric partial group $\mathbf{G}_{\mathbf{1}}$.

DEFINITION 2.8. Let $\mathbf{G}_{\mathbf{4}}$ be an extension of rank four of a symmetric partial group $\mathbf{G}_{\mathbf{1}}$ with $G_{0}, G_{1}, \ldots, G_{4}$ defined as before. Then we construct the semigroup $\mathbf{S}\left(\mathbf{G}_{1}, \mathbf{G}_{4}\right)$ on the set

$$
\left\{(i, g, j): 0<i \leq j \leq 5, g \in G_{j-i}\right\} \cup\{0\}
$$

with the multiplication $(i, g, j) \cdot(k, h, l)=(i, g h, l)$ if $j=k$ and $g h$ is the product of $g$ with $h$ in $\mathbf{G}_{\mathbf{4}}$ and 0 otherwise.

It is not difficult to verify that this is indeed a semigroup. Associativity holds essentially because we required it to be so in our definition of an extension of rank $k$. If $\mathbf{G}_{\mathbf{4}}$ is embeddable in a group $\mathbf{H}$, then $\mathbf{S}\left(\mathbf{G}_{1}, \mathbf{G}_{\mathbf{4}}\right)$ can be viewed as a subsemigroup of 'the upper half' of the Brandt semigroup $\mathbf{B}_{5}(\mathbf{H})$ over $\mathbf{H}$.

Let $\langle 1\rangle$ be the one element group. Now the set $B_{5}(\langle 1\rangle) \cap S\left(G_{1}, G_{4}\right)$ consists of those elements of the set $B_{5}(\langle 1\rangle)$ with the form $(i, 1, j)$ where $i \leq j$. Furthermore the restriction of the operations of both $\mathbf{B}_{5}(\langle\mathbf{1}\rangle)$ and $\mathbf{S}\left(\mathbf{G}_{\mathbf{1}}, \mathbf{G}_{4}\right)$ to this set coincide and form a subsemigroup. We will denote this subsemigroup by $\mathbf{S}(\langle\mathbf{1}\rangle,\langle\mathbf{1}\rangle)$ which is consistent with our previous definition since $\langle 1\rangle$ can be considered as an extension of rank four of itself. We can now construct the following amalgam.

DEFINITION 2.9. For a finite extension $\mathbf{G}_{\mathbf{4}}$ of rank four of a symmetric partial group $\mathbf{G}_{1}$ define an associated semigroup amalgam $A\left(\mathbf{G}_{\mathbf{1}}, \mathbf{G}_{\mathbf{4}}\right)$ by

$$
\mathbf{A}\left(\mathbf{G}_{\mathbf{1}}, \mathbf{G}_{\mathbf{4}}\right)=\left[\mathbf{S}\left(\mathbf{G}_{\mathbf{1}}, \mathbf{G}_{\mathbf{4}}\right), \mathbf{B}_{\mathbf{5}}(\langle\mathbf{1}\rangle) ; \mathbf{S}(\langle\mathbf{1}\rangle,\langle\mathbf{1}\rangle)\right] .
$$


The following tables representing $S\left(\mathbf{G}_{\mathbf{1}}, \mathbf{G}_{\mathbf{4}}\right)$ and $\mathbf{B}_{\mathbf{5}}(\langle\mathbf{1}\rangle)$ respectively may help to visualize the amalgam we have constructed (here $(i, G, j)=\{(i, g, j): g \in G\})$ :

\begin{tabular}{|c|c|c|c|c|}
\hline$\left(1, G_{0}, 1\right)$ & $\left(1, G_{1}, 2\right)$ & $\left(1, G_{2}, 3\right)$ & $\left(1, G_{3}, 4\right)$ & $\left(1, G_{4}, 5\right)$ \\
\hline & $\left(2, G_{0}, 2\right)$ & $\left(2, G_{1}, 3\right)$ & $\left(2, G_{2}, 4\right)$ & $\left(2, G_{3}, 5\right)$ \\
\hline & & $\left(3, G_{0}, 3\right)$ & $\left(3, G_{1}, 4\right)$ & $\left(3, G_{2}, 5\right)$ \\
\hline & & & $\left(4, G_{0}, 4\right)$ & $\left(4, G_{1}, 5\right)$ \\
\hline & & & & $\left(5, G_{0}, 5\right)$ \\
\hline
\end{tabular}

\begin{tabular}{|c|c|c|c|c|}
\hline$\left(1, G_{0}, 1\right)$ & $\left(1, G_{0}, 2\right)$ & $\left(1, G_{0}, 3\right)$ & $\left(1, G_{0}, 4\right)$ & $\left(1, G_{0}, 5\right)$ \\
\hline$\left(2, G_{0}, 1\right)$ & $\left(2, G_{0}, 2\right)$ & $\left(2, G_{0}, 3\right)$ & $\left(2, G_{0}, 4\right)$ & $\left(2, G_{0}, 5\right)$ \\
\hline$\left(3, G_{0}, 1\right)$ & $\left(3, G_{0}, 2\right)$ & $\left(3, G_{0}, 3\right)$ & $\left(3, G_{0}, 4\right)$ & $\left(3, G_{0}, 5\right)$ \\
\hline$\left(4, G_{0}, 1\right)$ & $\left(4, G_{0}, 2\right)$ & $\left(4, G_{0}, 3\right)$ & $\left(4, G_{0}, 4\right)$ & $\left(4, G_{0}, 5\right)$ \\
\hline$\left(5, G_{0}, 1\right)$ & $\left(5, G_{0}, 2\right)$ & $\left(5, G_{0}, 3\right)$ & $\left(5, G_{0}, 4\right)$ & $\left(5, G_{0}, 5\right)$ \\
\hline
\end{tabular}

\section{Proof of the main theorem and other results}

Theorem 1.4 and Theorem 1.5 now follow from the following theorem.

THEOREM 3.1. Let $\mathbf{G}_{1}$ be a symmetric partial group. The following are equivalent:

(1) $\mathrm{G}_{1}$ is embeddable in a group (finite group).

(2) There is an extension $\mathbf{G}_{\mathbf{4}}$ of rank four of $\mathbf{G}_{\mathbf{1}}$ that is embeddable in a group (finite group).

(3) There is an extension $\mathbf{G}_{\mathbf{4}}$ of rank four of $\mathbf{G}_{1}$ such that $\mathbf{A}\left(\mathbf{G}_{1}, \mathbf{G}_{\mathbf{4}}\right)$ is embeddable in an inverse semigroup (finite inverse semigroup).

(4) There is an extension $\mathbf{G}_{\mathbf{4}}$ of rank four of $\mathbf{G}_{1}$ such that $\mathbf{A}\left(\mathbf{G}_{1}, \mathbf{G}_{\mathbf{4}}\right)$ is embeddable in a semigroup (finite semigroup).

(5) There is an extension $\mathbf{G}_{\mathbf{4}}$ of rank four of $\mathbf{G}_{1}$ such that $\mathbf{A}\left(\mathbf{G}_{\mathbf{1}}, \mathbf{G}_{\mathbf{4}}\right)$ is weakly embeddable in a semigroup (finite semigroup).

PROOF. That (1) implies (2) follows from comments earlier in this paper (see before Lemma 2.5).

(2) implies (3): Say $\mathbf{G}_{\mathbf{4}}$ is embeddable in a group $\mathbf{H}$. Then it is easily verified that $A\left(G_{1}, G_{4}\right)$ is embedded in the Brandt semigroup $B_{5}(\mathbf{H})$ by the identity maps: $\nu_{1}: \mathbf{S}\left(\mathbf{G}_{1}, \mathbf{G}_{4}\right) \rightarrow \mathbf{B}_{5}(\mathbf{H})$ and $\nu_{2}: \mathbf{B}_{5}(\langle\mathbf{1}\rangle) \rightarrow \mathbf{B}_{5}(\mathbf{H})$ that take an element from their respective domains and assign to it the element with the same name in $\mathbf{B}_{\mathbf{5}}(\mathbf{H})$. Note that $\mathbf{B}_{5}(\mathbf{H})$ is finite if and only if $\mathbf{H}$ is a finite group and that $\mathbf{B}_{5}(\mathbf{H})$ is an inverse semigroup. 
(3) implies (4): Trivial.

(4) implies (5): This follows immediately from the fact that every embedding of an amalgam is a weak embedding of that amalgam.

(5) implies (1): Say the amalgam $A\left(\mathbf{G}_{\mathbf{1}}, \mathbf{G}_{\mathbf{4}}\right)$ is weakly embeddable in a semigroup $\mathbf{T}$ (a finite semigroup $\mathbf{T}$ ) via the injective homomorphisms $\nu_{1}: \mathbf{S}\left(\mathbf{G}_{\mathbf{1}}, \mathbf{G}_{\mathbf{4}}\right) \rightarrow \mathbf{T}$ and $\nu_{2}: \mathbf{B}_{5}(\langle\mathbf{1}\rangle) \rightarrow \mathbf{T}$.

For any element $g \in G_{1}$ consider the element $v_{1}(2, g, 3)$ of $\mathbf{T}$. Now since $\mathbf{G}_{\mathbf{1}}$ is symmetric, there is an element $g^{\prime}$ such that $g g^{\prime}=g^{\prime} g=1$ in $\mathbf{G}_{\mathbf{1}}$ (and of course in $\mathbf{G}_{4}$ since $\mathbf{G}_{1}$ is contained within $\left.\mathbf{G}_{4}\right)$. So $v_{1}(2, g, 3)\left[v_{1}\left(3, g^{\prime}, 4\right)\right]=v_{1}\left((2, g, 3)\left(3, g^{\prime}, 4\right)\right)=$ $v_{1}((2,1,4))$ and

$$
\begin{aligned}
v_{1}((2,1,4))\left[v_{2}((4,1,2)) v_{1}((2, g, 3))\right] \\
=\left[v_{1}((2,1,4)) v_{2}((4,1,2))\right] v_{1}((2, g, 3)) \\
=\left[v_{2}((2,1,4)) v_{2}((4,1,2))\right] \nu_{1}((2, g, 3)) \quad\left(\text { since } v_{1}((2,1,4))=v_{2}((2,1,4))\right) \\
=\left[v_{2}((2,1,4)(4,1,2))\right] v_{1}((2, g, 3)) \\
=\left[v_{2}((2,1,2))\right] v_{1}((2, g, 3)) \\
=\left[v_{1}((2,1,2))\right] v_{1}((2, g, 3)) \quad\left(\text { since } v_{1}((2,1,2))=v_{2}((2,1,2))\right) \\
=v_{1}((2, g, 3))
\end{aligned}
$$

Note that we do not know what the product $v_{2}((4,1,2)) v_{1}((2, g, 3))$ from the first line actually is in $\mathbf{T}$, only that it does exist. Therefore the set $H_{2,3}=\left\{\nu_{1}((2, g, 3)): g \in\right.$ $\left.G_{1}\right\}$ is $\mathscr{R}$-related to $\nu_{1}((2,1,4))$. In particular $H_{2,3}$ lies within an $\mathscr{R}$-class of $\mathbf{T}$.

Also $\left[v_{l}\left(\left(1, g^{\prime}, 2\right)\right)\right] v_{1}((2, g, 3))=v_{1}\left(\left(1, g^{\prime}, 2\right)(2, g, 3)\right)=v_{1}((1,1,3))$ and

$$
\begin{aligned}
& {\left[v_{1}((2, g, 3)) v_{2}((3,1,1))\right] v_{1}((1,1,3))} \\
& =v_{1}((2, g, 3))\left[v_{2}((3,1,1)) v_{1}((1,1,3))\right] \\
& =v_{1}((2, g, 3))\left[v_{2}((3,1,1)) \nu_{2}((1,1,3))\right] \\
& =v_{1}((2, g, 3))\left[v_{2}((3,1,1)(1,1,3))\right]=v_{1}((2, g, 3))\left[v_{2}((3,1,3))\right] \\
& =\nu_{1}((2, g, 3))\left[v_{1}((3,1,3))\right]=v_{1}((2, g, 3)) \text {. }
\end{aligned}
$$

Thus $H_{2,3}$ is within an $\mathscr{L}$-class of $\mathbf{T}$. In particular since $H_{2,3}$ is both $\mathscr{L}$-related and $\mathscr{R}$-related in $\mathbf{T}$, it lies within an $\mathscr{H}$-class of $\mathbf{T}$.

Now for each element $g \in G_{1}$ we can consider element $\nu_{1}((3, g, 4))$. Replacing every expression of the form $(i, h, j)$ in the above arguments by $(i+1, h, j+1)$ we obtain the analogous result that the set $H_{3,4}=\left\{(3, g, 4): g \in G_{1}\right\}$ is also contained in an $\mathscr{H}$-class of $\mathbf{T}$.

Consider the extension $\mathbf{G}_{2}$ of rank 2 of $\mathbf{G}_{1}$ consisting of the elements of $G_{2}$ with the partial operation $f \cdot g=h$ if and only if either $f$ or $g$ is contained in $G_{1}$ and $f g=h$ in $\mathbf{G}_{\mathbf{4}}$. We can construct the associated split system $\left(\{a\} \times G_{1} \times\{b\},\{b\} \times\right.$ 
$\left.G_{1} \times\{c\},\{a\} \times G_{2} \times\{c\}\right)$ and a corresponding embedding $(j, k, l)$ into $\mathbf{T}$ defined by $j((a, g, b))=v_{1}((2, g, 3)), k((b, g, c))=v_{1}((3, g, 4)), l((a, h, c))=v_{1}((2, h, 4))$

where $g$ is contained in $G_{1}$ and $h$ is contained in $G_{2}^{\prime}$. It is clear that these maps are injective and constitute an embedding of $\left(\{a\} \times G_{1} \times\{b\},\{b\} \times G_{1} \times\{c\},\{a\} \times G_{2}^{\prime} \times\{c\}\right)$ since $j((a, g, b)) k((b, h, c))=v_{1}((2, g, 3)) \nu_{1}((3, h, 4))=v_{1}((2, g, 3)(3, h, 4))=$ $\nu_{1}((2, g h, 4))=l((a, g h, c))$. Furthermore since the images of $j$ and $k$ are the sets $H_{2,3}$ and $H_{3,4}$ respectively and these lie within $\mathscr{H}$-classes of $\mathbf{T}$ we may apply Lemma 2.6 to show that $\mathbf{G}_{\mathbf{1}}$ is embeddable in a group. The theorem is proved.

More specifically we have proved

COROllarY 3.2. Given a semigroup amalgam $\mathbf{A}=\left[\mathbf{S}_{\mathbf{1}}, \mathbf{S}_{2} ; \mathbf{U}\right]$ with $\left|S_{2}\right| \leq 26$, $|U| \leq 16$, there is no algorithm to determine whether $\mathbf{A}$ is embeddable (or weakly embeddable) in any of the following: a semigroup; a finite semigroup; an inverse semigroup; a finite inverse semigroup. As $\mathbf{S}_{2}$ we may take $\mathbf{B}_{5}(\langle\mathbf{1}\rangle)$ and as $\mathbf{U}$ we may take $\mathbf{S}(\langle\mathbf{1}\rangle,\langle\mathbf{1}\rangle)$.

So the weak decision problem for amalgam embeddability in the class of inverse semigroups and finite inverse semigroups is undecidable.

In the case of embedding (weak or otherwise) a semigroup amalgam in a finite semigroup (or in a finite inverse semigroup) we may improve the bounds in this theorem as follows.

THEOREM 3.3. Given a semigroup amalgam $\mathbf{A}=\left[\mathbf{S}_{1}, \mathbf{S}_{2} ; \mathbf{U}\right]$ with $\left|S_{2}\right| \leq 7$, $|U| \leq 5$, there is no algorithm to determine whether $\mathbf{A}$ is embeddable in a finite semigroup or a finite inverse semigroup.

PROOF. This essentially follows from the main result in [8]. For any extension $\mathbf{G}_{\mathbf{3}}$ of rank 3 of a partial group $\mathbf{G}_{1}$ (with $G_{i}$ for $i \leq 3$ defined as before) we may construct a semigroup $S\left(G_{1}, G_{3}\right)$ in the following way: the universe of $\mathbf{S}\left(\mathbf{G}_{\mathbf{1}}, \mathbf{G}_{\mathbf{3}}\right)$ is the set $\left\{(i, g, j): 0<i<j \leq 4, g \in G_{j-i}\right\}$ and the multiplication is defined in the same way as that for $\mathbf{S}\left(\mathbf{G}_{\mathbf{1}}, \mathbf{G}_{\mathbf{4}}\right)$ in Definition 2.8 (this semigroup first appeared in [3]). In [8], Kublanovsky and Sapir show that for a symmetric partial group $\mathbf{G}_{\mathbf{1}}$ one can find an extension $\mathbf{G}_{\mathbf{3}}$ of rank three of $\mathbf{G}_{\mathbf{1}}$ embeddable in a finite group, if and only if one can find a finite semigroup $\mathbf{T}$ containing $\mathbf{S}\left(\mathbf{G}_{1}, \mathbf{G}_{3}\right)$ with elements $x, y \in T^{1}$ such that $x \cdot(1,1,4) \cdot y=(2,1,3)$ in $\mathbf{T}$. With this in mind, we can construct an amalgam consisting of $\mathbf{S}\left(\mathbf{G}_{\mathbf{1}}, \mathbf{G}_{\mathbf{3}}\right)$ along with a semigroup that enforces this condition in any embedding semigroup. This second semigroup, $\mathbf{S}_{\mathbf{2}}$, can be taken as the set

$$
\{(2,1,1),(4,1,3),(2,1,3),(1,1,4),(2,1,4),(1,1,3), 0\}
$$


with multiplication as within a Brandt semigroup. The set

$$
U=\{(2,1,3),(1,1,4),(2,1,4),(1,1,3), 0\}
$$

is common to both $S_{2}$ and $\mathbf{S}\left(\mathbf{G}_{\mathbf{1}}, \mathbf{G}_{\mathbf{3}}\right)$ and furthermore the restriction of the operations of these semigroups to $U$ coincide and forms a subsemigroup of both which we will call $\mathbf{U}$. It is now easily verified that the following constitutes a semigroup amalgam:

$$
A^{\prime}\left[G_{1}, G_{3}\right]=\left[S\left(G_{1}, G_{3}\right), S_{2} ; U\right] .
$$

Furthermore if $\mathbf{G}_{\mathbf{3}}$ is embeddable in a finite group $\mathbf{H}$, then this amalgam is embeddable in $B_{4}(H)$ in the obvious way (analogous to the embedding of $A\left(G_{1}, G_{4}\right)$ into $B_{5}(H)$ ). On the other hand, if $A^{\prime}\left(\mathbf{G}_{\mathbf{1}}, \mathbf{G}_{\mathbf{3}}\right)$ is embeddable into a finite semigroup $\mathbf{T}$ by the maps $v_{1}$ and $v_{2}$ then we have

$$
\begin{aligned}
v_{2}((2,1,1)) v_{1}((1,1,4)) \nu_{2}((4,1,3)) & =v_{2}((2,1,1)) v_{2}((1,1,4)) v_{2}((4,1,3)) \\
& =v_{2}((2,1,3))=v_{1}((2,1,3))
\end{aligned}
$$

and therefore $\mathbf{G}_{\mathbf{1}}$ is embeddable in a group.

Note that there is a subsemigroup $\mathbf{U}^{\prime}$ of $\mathbf{U}$ on the three element set

$$
\{(2,1,3),(1,1,4), 0\}
$$

If we replace $\mathbf{U}$ by $\mathbf{U}^{\prime}$ throughout the proof of Theorem 3.3, all arguments remain valid except the natural embedding of $\mathbf{A}^{\prime}\left(\mathbf{G}_{\mathbf{1}}, \mathbf{G}_{\mathbf{3}}\right)$ into $\mathbf{B}_{\mathbf{4}}(\mathbf{H})$ is now only a weak amalgam embedding since $\nu_{1}((2,1,4))=v_{2}((2,1,4))$ though $(2,1,4) \notin U^{\prime}$. Thus we have proved

THEOREM 3.4. Given a semigroup amalgam $\mathbf{A}=\left[\mathbf{S}_{1}, \mathbf{S}_{2} ; \mathbf{U}\right]$ with $\left|S_{2}\right|=7$, $|U|=3$, there is no algorithm to determine whether $\mathbf{A}$ is weakly embeddable in a finite semigroup.

In [12], Sapir proves the undecidablity of the strong decision problem for amalgam embeddability in the class of finite semigroups using an almost identical structure to that we use to prove Theorem 3.3 above however the bounds for $\left|S_{2}\right|$ and $|U|$ are 17 and 7 respectively.

Fundamental to the proof of Kublanovsky and Sapir's result [8] is the fact that finite semigroups consisting of only one non zero $\mathscr{J}$-class have a particularly well defined structure: they are completely 0 -simple and by a well known theorem of Rees, isomorphic to a Rees Matrix semigroup with zero over a group (see [1] or [5] for details). The completely 0 -simple structure is not available in the general case of embedding in a $\mathscr{J}$-class of an arbitrary semigroup (indeed any finite semigroup can 
be embedded in an infinite semigroup with a single $\mathscr{J}$-class which is not completely 0 -simple), and this is why the proofs of Theorem 3.3 and Theorem 3.4 only apply for embedding amalgams in the class of finite semigroups.

NOTE 3.5. Theorem 3.2, Theorem 3.3 and Theorem 3.4 have ring analogues. To obtain these we can replace 'semigroup' with 'ring' and any numbers $n$ appearing in the theorems by $2^{n}$.

This is because if $\mathbf{S}$ is a finite semigroup with $n$ elements then the semigroup ring $\mathbb{Z}_{2}[\mathbf{S}]$ has $2^{n}$ elements.

Necessary and sufficient conditions for the embeddability of a semigroup amalgam into a semigroup have been found by Howie [4]. We will describe this characterisation since by Theorem 1.4 the conditions involved must not be algorithmically verifiable.

Let $\mathbf{A}=\left[\left\{\mathbf{S}_{\mathbf{i}}: i \in I\right\} ; \mathbf{U} ;\left\{\phi_{i}: i \in I\right\}\right]$ be a semigroup amalgam. We will assume that the sets $S_{i}$ are pairwise disjoint. The free product, $\Pi^{*} \mathbf{S}_{\mathbf{i}}$, is the semigroup generated by $X_{A}=\cup S_{i}$ with the Cayley tables of the $S_{\mathbf{i}}$ as relations $R_{A}$. That is, $\Pi^{*} \mathbf{S}_{\mathbf{i}}$ is the semigroup $\left\langle X_{A} ; R_{A}\right\rangle$. We may define a congruence $\theta$ on $\Pi^{*} S_{i}$ as the congruence generated by $\left\{\left(\phi_{i}(u), \phi_{j}(u)\right): i, j \in I, u \in U\right\}$. The free product of the amalgam $\mathbf{A}$ is the semigroup $\Pi_{U}^{*} S_{\mathrm{i}}=\left\langle X_{A} ; R_{A}\right\rangle / \theta$. For each $i \in I$ there are homomorphisms $v_{i}$ from each $\mathbf{S}_{\mathbf{i}}$ into $\Pi_{U}^{*} \mathbf{S}_{\mathbf{i}}$ defined by $v_{i}(s)=s$. If these maps constitute an embedding of the amalgam $\mathbf{A}$, then it is said that $\mathbf{A}$ is naturally embedded in its free product.

THEOREM 3.6 ([4]). The amalgam $\mathbf{A}$ is embeddable in a semigroup if and only if it is naturally embedded in its free product.

Let $X_{A}^{\prime}$ be the set

$$
U \cup\left(X_{A} \backslash\left\{\phi_{i}(u): u \in U, i \in I\right\}\right)
$$

and $R_{A}^{\prime}$ the set of Cayley tables of the $S_{\mathrm{i}}$ with every occurrence of an element of the form $\phi_{i}(u)$ replaced by the element $u$. We have

$$
\Pi_{U}^{*} \mathrm{~S}_{\mathrm{i}}=\left\langle X_{A} ; R_{A}\right\rangle / \theta \cong\left\langle X_{A}^{\prime} ; R_{A}^{\prime}\right\rangle .
$$

The previous theorem can now be restated as

THEOREM 3.7. The amalgam $\mathbf{A}$ is embeddable in a semigroup if and only if the elements $X_{A}^{\prime}$ are distinct in $\left\langle X_{A}^{\prime} ; R_{A}^{\prime}\right\rangle$.

Thus we may reformulate Theorem 1.4 for semigroups as

COROLLARY 3.8. There is no algorithm that will solve the following decision problem: given a finite semigroup amalgam $\mathbf{A}$, determine whether two generators $x, y \in X_{A}$ represent different elements of the semigroup $\left\langle X_{A}^{\prime} ; R_{A}^{\prime}\right\rangle$. 
We finish with some questions.

QUESTION 3.9. (i) What are the minimal pairs $\left(\left|S_{2}\right|,|U|\right)$ for which Theorem 3.2, Theorem 3.3 or Theorem 3.4 (or their ring analogues) are true and are these minimal pairs the same?

(ii) Are there classes for which the decision problem for amalgam embeddability is decidable (or undecidable) and the decision problem for weak amalgam embeddability is undecidable (or decidable respectively)?

(iii) Are there varieties $V$ for which the (strong or weak) decision problem for amalgam embeddability or weak amalgam embeddability is decidable (or undecidable) but the opposite is true for the finite trace of $V$ (that is, the finite members of $V$ )?

Regarding the first of these questions we note that in [2] it is shown that any semigroup amalgam with a two element core is embeddable in a semigroup. The last question seems of particular interest when $V$ is the class of inverse semigroups (which form a variety in the signature $\left\{\cdot,^{-1}\right\}$ ) since it is known that every inverse semigroup amalgam is embeddable in an inverse semigroup, but also that not every finite inverse semigroup amalgam is embeddable in a finite inverse semigroup (see [5]). We note however that one of the main results of [10] shows that there is an algorithm that determines, given a finite semigroup amalgam $\mathbf{A}$ with inverse semigroup core, whether $\mathbf{A}$ is embeddable in a finite semigroup, though the embedding semigroup is not inverse.

NOTE 3.10. (Added in proof.) Denote the 14 element subsemigroup of $\mathbf{B}_{5}(\langle\mathbf{1}\rangle)$ on the elements: $\{(1,1,1),(1,1,3),(1,1,5),(2,1,2),(2,1,4),(3,1,3),(3,1,5)$, $(3,1,1),(4,1,4),(4,1,2),(5,1,5),(5,1,3),(5,1,1), 0\}$ by $\mathbf{C}_{5}$. For any extension $\mathbf{G}_{\mathbf{4}}$ of rank four of a symmetric partial group $G_{1}$, the semigroup $S\left(G_{1}, G_{4}\right)$ intersects $C_{5}$ on a 10 element subsemigroup, $V$ say. If we replace the amalgam $A\left(G_{1}, G_{4}\right)$ by $\left[\mathbf{S}\left(\mathbf{G}_{1}, \mathbf{G}_{4}\right), \mathbf{C}_{5} ; \mathbf{V}\right]$ throughout, the proof of Theorem 3.1 continues to be valid and we obtain a new version of Corollary 3.2 with bounds of 14 and 10 respectively. If we replace the core semigroup $\mathbf{V}$ in this construction by the 8 element subsemigroup on $\{(1,1,3),(1,1,5),(2,1,2),(2,1,4),(3,1,3),(3,1,5),(4,1,4), 0\}$, then (as in the comments preceding Theorem 3.4 ) we obtain a further improved bound in the case of weak amalgam embeddability in the class of all semigroups (this time 14 and 8 ).

\section{References}

[1] A. H. Clifford and G. B. Preston, The algebraic theory of semigroups, Volumes 1, 2, (American Mathematical Society, Providence, RI, 1961, 1967). 
[2] T. E. Hall, 'Representation extension and amalgamation for semigroups', Quart. J. Math. Oxford (2) 29 (1978), 309-334.

[3] T. Hall, S. Kublanovsky, S. Margolis, M. Sapir and P. Trotter, 'Decidable and undecidable problems related to finite 0-simple semigroups', J. Pure Appl. Algebra 119 (1997), 75-96.

[4] J. M. Howie, 'An embedding theorem with amalgamation for semigroups', Proc. London Math. Soc. (3), 12 (1962), 511-534.

[5] — Fundamentals of semigroup theory, 2nd edition (Oxford University Press, New York, 1995).

[6] M. G. Jackson, 'Some undecidable embedding problems for finite semigroups', Proc. Edinburgh Math. Soc. (II) 42 (1999) 113-125.

[7] O. G. Kharlampovich and M. V. Sapir, 'Algorithmic problems in varieties', Internat. J. Algebra Comput. 5 (1995), 379-602.

[8] S. Kublanovsky and M. V. Sapir, 'Potential divisibility in finite semigroups is undecidable', Internat. J. Algebra Comput. 8 (1998), 671-679.

[9] _ 'A variety where the set of subalgebras of finite simple algebras is not recursive', Internat. J. Algebra Comput. 8 (1998), 680-688.

[10] J. Okniński and M. S. Putcha, 'Embedding finite semigroup amalgams', J. Austral. Math. Soc. (Series A) 51 (1991), 489-496.

[11] M. V. Sapir, 'Eventually $\mathscr{H}$-related sets and systems of equations over finite semigroups and rings', J. Algebra 183 (1996), 365-377.

[12] _ 'Algorithmic problems for amalgams of finite semigroups', to appear in J. Algebra.

[13] O. Schreier, 'Die Untergruppen der freien Gruppen', Abhandlungen aus dem mathematischen Seminar der Hansischen Universität Hamburg 5 (1927), 161-183.

\section{Discipline of Mathematics}

University of Tasmania

GPO Box 252-37

Hobart 7001

Australia

email: marcel_j@hilbert.maths.utas.edu.au

now at

Department of Mathematics

La Trobe University

Bundoora VIC 3083

Australia

e-mail: m.g.jackson@latrobe.edu.au 